\title{
Formulation and evaluation of an automatic dishwashing detergent containing $\mathrm{T} 1$ lipase
}

\begin{abstract}
The use of enzymes in detergent formulations is becoming popular due to the concerns about the environment. T1 lipase (E.C. 3.1.1.3) was evaluated for its stability and performance in dishwashing along with other common components of an automatic dishwashing detergent. Therefore, the process of formulating the detergent would depend on the stability of T1 lipase, which may also reflect the performance during the washing. T1 lipase was mostly stable in nonionic surfactants, especially those that were made of polyhydric alcohols. T1 lipase was also stable in a mixture of sodium carbonate and glycine. However, sodium carbonate alone destabilized T1 lipase possibly due to the interaction between carbonates and $\mathrm{Ca} 2+$. These results indicated that polyhydric alcohols and glycine had stabilizing effects on T1 lipase. The dishwashing performance was evaluated in term of percent soil removed. The dishwashing performance of the formulated detergent was positively affected by the increase in temperature but negatively affected by the presence of hard water, specifically $\mathrm{Ca} 2+$ and $\mathrm{Mg} 2+$. However, T1 lipase was not negatively affected by the presence of hard water, and this enzyme was enhanced by the presence of polyacrylates. The presence of $\mathrm{Ca} 2+$ improved the structural integrity of T1 lipase. It is generally known that most enzymes that depend on $\mathrm{Ca} 2+$ for their structural integrity would be greatly destabilized in the presence of metal chelators; thus, stabilizing strategies such as adding glycine would be essential to maintain enzyme activity during the wash.
\end{abstract}

Keyword: Lipase; Thermostable; Detergent; Surfactant; Dishwashing analysis; Hard water; Enzyme stabilizer 